\title{
Influencia del liderazgo directivo y la motivación de trabajadores, Unidad de Gestión Educativa Local Huaraz
}

Influence of managerial leadership and motivation of workers, Huaraz Local Education Management Unit

Elena Ramírez Baltazar, Julio C. Bustamante Cabello', William J. Marín Rodriguez, Dalila I. Villanueva Cadenas ${ }^{2}$, Flor Lioo Jordán², Walter S. Gil Quevedo², Luis A. Baldeos Ardían², Cesar F. Gaviria Henriquez², Armando A.

Pesantes de la Cruz

\section{RESUMEN}

Objetivo: Determinar la influencia del liderazgo directivo en la motivación de los trabajadores de la Unidad de Gestión Educativa Local Huaraz año 2017. Material y Métodos: El tipo de estudio fue correlacional causal, con un diseño de investigación no experimental, la población conformada por 54 trabajadores, a quienes se les aplicó un cuestionario para recoger información de las variables de estudio. Resultados: Los resultados obtenidos determinaron la influencia del liderazgo directivo en la motivación de los trabajadores de la Unidad de Gestión Educativa Local Huaraz año 2017, se realizaron las pruebas de hipótesis general y específicas, se determinó que el liderazgo directivo influye significativamente en la motivación de los trabajadores en la Unidad de Gestión Educativa Local Huaraz, se analizó la influencia del liderazgo directivo en la motivación de los trabajadores, mediante la prueba ETA, obteniendo los resultados de 0.805 para liderazgo directivo y 0.562 para motivación de los trabajadores, lo cual afirma la hipótesis alternativa el liderazgo directivo tiene influencia significativa en la motivación de los trabajadores de la Unidad de Gestión Educativa Local Huaraz año 2017. Conclusión: Existe influencia significativa entre el liderazgo directivo y la motivación de los trabajadores, esto se explica por qué los directivos de la Unidad de Gestión Educativa Local Huaraz con frecuencia aplicaron diversas estrategias de liderazgo directivo en la motivación de sus trabajadores en la consecución de los objetivos institucionales.

Palabras clave: Liderazgo directivo, motivación de trabajadores, reconocimiento público, conducta responsable, persistencia de trabajadores.

\section{ABSTRACT}

Objective: To determine the influence of managerial leadership on the motivation of the workers of the Huaraz Local Education Management Unit 2017. Material and Methods: The type of study was correlational - causal, with a non-experimental research design, the population formed by 54 workers, to whom a questionnaire was applied to collect information on the study variables. Results: The results obtained determined the influence of the leadership leadership in the motivation of the workers of the Huaraz Local Education Management Unit 2017, the tests of general and specific hypotheses were performed, it was determined that the leadership leadership significantly influences the motivation of In the Huaraz Local Education Management Unit, the influence of managerial leadership on the motivation of workers was analyzed, using the ETA test, obtaining the results of 0.805 for leadership leadership and 0.562 for motivation of workers, which affirms the alternative hypothesis the leadership leadership has significant influence on the motivation of the workers of the Huaraz Local Education Management Unit 2017. Conclusion: There is significant influence between the managerial leadership and the motivation of the workers, this explains why the managers of the Educativ Management Unit a Local Huaraz frequently applied various managerial leadership strategies in motivating their workers to achieve institutional objectives.

Keywords: Directive leadership, motivation of workers, public recognition, responsible behavior, persistence of workers

\footnotetext{
${ }^{1}$ Universidad Cesar Vallejo. Lima, Perú

${ }^{2}$ Universidad Nacional José Faustino Sánchez Carrión. Huacho, Perú

${ }^{3}$ Universidad San Pedro. Chimbote, Perú
} 


\section{INTRODUCCIÓN}

Actualmente en la organizaciones los directivos utilizan diversas estrategias para motivar a sus trabajadores, sin embargo también se presentan problemas en que la mayoría de veces no se toma en cuenta, como son el sentimiento y pensamiento de los trabajadores, algunos directivos expresan escasa preocupación por ayudarlos y apoyarlos para que puedan realizar las funciones laborales y sentir que valoran el esfuerzo que aportan para elevar su desempeño laboral; a veces no prestan atención a la motivación que los empleados exigen de forma reprimida o pasiva, por el contrario, se enfocan en presionar en el cumplimiento de objetivos y el logro de resultados, para ello exigen autoritariamente explotar sus habilidades, capacidades y competencias sin una adecuada motivación sea intrínseca o extrínseca.

En este contexto Las Unidades de Gestión Educativa Local no son ajenas a esta realidad, conformados por directores, quienes tienen la tareas de ejercer y aplicar el liderazgo directivo en el desempeño de sus funciones, que lideren los procesos funcionales, encontrar o visionar futuros problemas educativos, emplear mecanismos de motivación a sus empleados con la finalidad de que ellos puedan sentirse parte de la institución, sin embargo de acuerdo con los estudios e investigaciones realizadas, se desconoce el tipo de liderazgo directivo que estas autoridades utilizan, se desconoce si el tipo de liderazgo aplicado influye en la motivación de sus trabajadores, si los directores realizan acciones de motivación laboral a sus trabajadores y si los directores conocen que la motivación es un elemento muy importante en la gestión de estas instituciones.

Jaime (2015), en su tesis titulada "Estudio del liderazgo de docentes y directivos en programas técnicos tecnológicos de la universidad cooperativa de Colombia, de Bucaramanga. Colombia", realizada en la Universidad de Granada, el objetivo general es estudiar el liderazgo de los docentes en los programas técnicos tecnológicos de la Universidad Cooperativa de Colombia; concluyendo: que los estilos de liderazgo que existen en los docentes y directivos de los programas técnicos y tecnológicos profesionales de la Universidad Cooperativa son transformacionales, transaccionales y correctivo/evitador. Estableciendo que el liderazgo transformacional predomina sobre el transaccional y el correctivo evitador. Cada estilo de liderazgo se identifica con un número de actitudes, comportamientos y prácticas que lo definen. La mayor frecuencia de estilo de liderazgo es el transformacional ya que garantiza la práctica de los líderes basados en la estimulación individual, el carisma y la motivación, de igual forma el estilo transaccional motiva a la recompensa por resultado, sin embargo, el ejercicio de su práctica al ser intermitente no garantiza que éstos contribuyan plenamente a una gestión educativa planificada. El liderazgo transformacional influye en los seguidores (estudiantes), posibilita el cambio organizacional.

Los líderes retroalimentan sus aptitudes y comportamientos de acuerdo a la aceptación o resistencia. Por ser el liderazgo con mayor presencia en las prácticas de los docentes/directivos, permite establecer cambios y transformaciones organizacionales, propicia y motiva el desarrollo de los estudiantes, de forma individual; incentiva imaginarios de visión, misión colectiva.

Quispe (2011), en su investigación denominada "Relación entre el estilo de liderazgo del director y el desempeño docente en las instituciones educativas públicas del 2do. sector de Villa El Salvador de la UGEL 01 San Juan de Miraflores, en los años 2009 y 2010", realizada en la Universidad Nacional Mayor de San Marcos de Lima Perú; se evidencia que existe relación débil entre el estilo de liderazgo autoritario del director con el desempeño docente y alumnos y, como tal, este estilo de liderazgo es el que más prevalece e interviene en el desempeño docente, los otros estilos como, el liderazgo democrático del director ha contribuido positivamente en las dimensiones: capacidades pedagógicas, responsabilidad en el desempeño de sus funciones laborales y las relaciones interpersonales con sus estudiantes, y los estilos autoritario y liberal han contribuido negativamente en la labor educativa del docente, estudio realizado a una muestra de 156 personas entre estudiantes y docentes.

Reyes (2012), en su investigación titulada "Liderazgo directivo y desempeño docente en el nivel secundario de una Institución Educativa de Ventanilla - Callao", realizada en la Escuela de Postgrado de la Universidad San Ignacio de Loyola Lima, se observa que no existe relación significativa entre la percepción del liderazgo directivo y el desempeño docente en el nivel secundario de una institución educativa del distrito de Ventanilla Callao, tampoco existe relación con los diferentes tipos de liderazgo estudiados con el desempeño docente, evidenciándose en esta investigación descriptiva la confirmación de la hipótesis nula, para ello se trabajó con una muestra de estudio de 40 docentes de educación secundaria de la institución educativa.

Sum (2015), en su investigación titulada "Motivación y desempeño laboral", realizada en la Universidad Rafael Landívar de México, el objetivo es determinar la influencia de la motivación en el 
desempeño laboral en el personal administrativo de la empresa de alimentos de la zona 1 de Quetzaltenango. La investigación es de tipo descriptivo correlacional, con una población compuesta por treinta y cuatro (34) colaboradores (personal administrativo) de la empresa de alimentos de la zona 1 de Quetzaltenango, son 12 mujeres y 22 hombres comprendidos entre las edades de 18 a 44 años, concluye que la motivación influye en el desempeño laboral del personal administrativo de la empresa de alimentos de la zona 1 de Quetzaltenango, con la aplicación de una prueba estandarizada que mide la escala de motivaciones psicosociales que miden el nivel de motivación, para ellos se evalúa diferentes factores, como la aceptación e integración social, reconocimiento social, autoestima, auto concepto, autodesarrollo, poder, seguridad, obteniéndose diferentes puntuaciones en su mayoría altas en base a dos aspectos el incentivo y la satisfacción.

La investigación alcanza su justificación para determinar la influencia del liderazgo directivo en la motivación de los trabajadores de la Unidad de Gestión Educativa Local Huaraz año 2017 y en función a ella, los directivos tomen las decisiones acertadas en beneficio de la institución, los principales beneficiarios son los trabajadores por que afecta de forma positiva en la motivación de los trabajadores. El objetivo de la presente investigación es determinar la influencia del liderazgo directivo en la motivación de los trabajadores de la Unidad de Gestión Educativa Local Huaraz año 2017.

\section{MATERIALES Y MÉTODOS}

El tipo de investigación fue no experimental, no se realizó manipulación o intervención en el tratamiento de las variables del estudio. La investigación fue transeccional debido a que se recolecto información de los indicadores de las variables de estudio en un tiempo determinado en un solo momento (Hernández, Fernández y Baptista, 2010). El diseño de la investigación fue correlacional causal porque se tuvo que determinar la influencia del liderazgo directivo en la motivación de los trabajadores de la Unidad de Gestión Educativa Local Huaraz año 2017.

La población de estudio estuvo conformada por 54 trabajadores tomados del cuadro de asignación de personal de la Unidad de Gestión Educativa Local Huaraz en el año 2017, siendo la muestra la misma población de estudio. Se utilizó la encuesta y cuestionario como técnica e instrumento de recolección de datos respectivamente. Se usó la escala ordinal para medir las variables de liderazgo directivo y la motivación de los trabajadores, con rangos y así asignarles un nivel respectivo (deficiente, eficiente y regular) para ambas variables.

Tabla 1. Escala de medición para variables de estudio.

\begin{tabular}{lcc}
\multicolumn{1}{c}{ Variable } & Nivel & Puntuación \\
\hline \multirow{2}{*}{ Liderazgo directivo } & Deficiente & $3-4$ \\
& Regular & $8-12$ \\
& Eficiente & $13-15$ \\
\hline Motivación de los trabajadores & Deficiente & $4-8$ \\
& Regular & $9-12$ \\
& Eficiente & $13-16$ \\
\hline
\end{tabular}

Antes de la aplicación del instrumento (cuestionario), fue validada por juicios de expertos con la finalidad de medir la relación entre las variables de estudio, se determinó su confiabilidad mediante Alfa de Cronbach (coeficiente que sirve para medir la fiabilidad del instrumento), se utilizó el cuestionario Serqvual, ya que es un cuestionario validado mediante la escala Likert. (Hernández, Fernández, Baptista, 2010), el resultado que se obtuvo mediante la aplicación del alfa de Cronbach fue de 0.83 , lo cual indica que hay una alta fiabilidad en el instrumento de recolección de datos. Con respecto al método de análisis de datos, fue el método observacional, analítico, sintético, inductivo y deductivo.

Los instrumentos fueron registrados en Microsoft Excel, los datos fueron registrados en función de cada pregunta por indicadores, cada indicador por dimensiones, y las dimensiones en función de las variables de investigación, posteriormente se crearon las tablas de frecuencias por cada pregunta y se hizo el resumen por cada dimensión. Asimismo, se aplicó la estadística 
descriptiva, para conseguir resultados porcentuales y frecuencias plasmadas en tablas y gráficas.

Se utilizó el software estadístico SPSS V.22.0 para las tablas de frecuencias en función de los rangos de valores obtenidos en la investigación. Posteriormente se hicieron los cruces de los datos de las tablas para el objetivo general y los objetivos específicos. Se interpretó las tablas estadísticas, citando de acuerdo con la norma APA vigente. Se realizó la prueba de hipótesis ETA, que es una medida de asociación cuyo valor siempre está comprendido entre 0 y 1 . El valor 0 indica que no hay asociación entre las variables de fila y de columna. Los valores cercanos a 1 indican que hay gran relación entre las variables. ETA resulta apropiada para una variable dependiente medida en una escala de intervalo y una variable independiente con un número limitado de categorías. Se calculan dos valores ETA: para las variables filas y columnas como una variable de intervalo.

\section{RESULTADOS}

La Tabla 2, demuestra que el liderazgo directivo tuvo influencia en la motivación de los trabajadores de la institución, con un valor del Coeficiente de ETA de 0,805 y 0,562 para cada variable respectivamente.

Tabla 2. Prueba ETA con respecto a la influencia del liderazgo directivo en la motivación de los trabajadores.

\begin{tabular}{|c|c|c|c|}
\hline & & & Valor \\
\hline \multirow[b]{2}{*}{ Nominal por intervalo } & \multirow[b]{2}{*}{ ETA } & $\begin{array}{l}\text { Liderazgo } \\
\text { Directivo }\end{array}$ & 0,805 \\
\hline & & $\begin{array}{l}\text { Motivación de los } \\
\text { trabajadores }\end{array}$ & 0,562 \\
\hline
\end{tabular}

La Tabla 3, demuestra que el liderazgo directivo tuvo influencia en el reconocimiento público de la función de los trabajadores de la institución, con un valor del Coeficiente de ETA de 0,565 y 0,383 para cada variable respectivamente.

Tabla 3. Prueba ETA con respecto a la influencia del liderazgo directivo en el reconocimiento público de la función de los trabajadores.

\begin{tabular}{lllc} 
& & Valor \\
Nominal por intervalo & ETA & $\begin{array}{l}\text { Reconocimiento } \\
\text { público de la función }\end{array}$ & 0,565 \\
\hline
\end{tabular}

La Tabla 4, demuestra que el liderazgo directivo tuvo influencia en la conducta responsable de los trabajadores de la institución, con un valor del
Coeficiente de ETA de 0,661 y 0,511 para cada variable respectivamente.

Tabla 4. Prueba ETA con respecto a la influencia del liderazgo directivo en la conducta responsable de los trabajadores.

\begin{tabular}{lccc} 
& Liderazgo Directivo & Valor \\
\hline Nominal por intervalo & ETA & $\begin{array}{l}\text { Conducta responsable } \\
\text { de los trabajadores }\end{array}$ & 0,511 \\
\hline
\end{tabular}

La Tabla 5, demuestra que el liderazgo directivo tuvo influencia en la persistencia de los trabajadores de la institución, con un valor del
Coeficiente de ETA de 0,693 y 0,536 para cada variable respectivamente. 
Tabla 5. Prueba ETA con respecto a la influencia del liderazgo directivo en la persistencia de los trabajadores.

\begin{tabular}{|c|c|c|c|}
\hline & & & Valor \\
\hline & & Liderazgo Directivo & 0,693 \\
\hline Nominal por intervalo & ETA & $\begin{array}{l}\text { Persistencia de los } \\
\text { trabajadores }\end{array}$ & 0,536 \\
\hline
\end{tabular}

\section{DISCUSIÓN}

Los resultados encontrados en la investigación permiten comparar con otras investigaciones similares:

Jaime (2015), en su investigación confirma que el estilo de liderazgo de los docentes se relaciona con las actitudes, las conductas y las buenas prácticas de los trabajadores, el liderazgo influye en la motivación y esta se orienta hacia la concreción del cambio organizacional. Esto encuentra su explicación en que el liderazgo aplicado retroalimenta las conductas, las aptitudes y los comportamientos de los trabajadores en función del acuerdo, aceptación o rechazo hacia el liderazgo y que el liderazgo de los directivos permite establecer cambios y transformaciones organizacionales, ayuda y motiva el desarrollo de quienes se aplica el liderazgo directivo.

Reyes (2012), en su investigación concluye que no existió relación significativa entre la percepción del liderazgo autoritario benevolente y el desempeño docente, no existió relación significativa entre la percepción del liderazgo consultivo y el desempeño docente en el nivel secundario de una institución educativa del distrito de Ventanilla - Callao 2009, no se cumplieron las hipótesis específicas ni la general, por lo que difieren significativamente con la investigación realizada.

Quispe (2011), en su investigación concluyó que existió influencia débil de 0,4675 entre el estilo de liderazgo autoritario del director y el desempeño docente, siendo influencia débil que representa el 0,4854 con los alumnos en el estilo de liderazgo que prevaleció en el desempeño docente, mientras que las relaciones encontradas en la presente investigación fueron de influencia mediana de 0,805 y 0,562, por lo que difiere significativamente en las conclusiones obtenidas en la investigación realizada.

Sum (2015), concluye que la motivación tiene la capacidad de influir positivamente en la otra variable, por lo que los resultados de su investigación coinciden con las conclusiones obtenidas en la investigación realizada.

La investigación en función a los resultados obtenidos se concluye que existe influencia significativa del liderazgo directivo sobre la motivación y el reconocimiento público de la función de los trabajadores. Este nivel de influencia se explica por qué los directivos de la Unidad de Gestión Educativa Local Huaraz con mediana frecuencia han motivado y reconocido las funciones realizadas por los trabajadores.

Con respecto al liderazgo sobre la conducta responsable se caracterizó por tener influencia. La característica de este nivel de influencia se explica por qué el liderazgo directivo de las autoridades de la Unidad de Gestión Educativa Local Huaraz conllevó a conductas responsables por parte de los trabajadores de la institución. Asimismo, se observó la influencia del liderazgo sobre la persistencia de los trabajadores. La característica de este nivel de influencia se explica por qué el liderazgo que presentan los directivos de la Unidad de Gestión Educativa Local Huaraz motivó a que los trabajadores persistan en el logro de sus objetivos.

\section{AGRADECIMIENTOS}

Al equipo colaborador por su aporte significativo en el presente artículo científico.

\section{REFERENCIAS BIBLIOGRÁFICAS}

Jaime, M. (2015). "Estudio del liderazgo de docentes y directivos en programas técnicos tecnológicos de la universidad cooperativa de Colombia, de Bucaramanga- Colombia". Tesis doctoral. Bucaramanga, Colombia.

Hernández, R, Fernández, C. \& Baptista, P. (2010). Metodología de la Investigación. (Quinta edición). México. D.F.: Mc Graw Hill.

Quispe, P. (2011). Relación entre el estilo de liderazgo del director y el desempeño docente en las instituciones educativas públicas del 2do. Sector de Villa El Salvador de la UGEL 01 San Juan de Miraflores, en los años 2009 y 2010. Tesis para optar el 
grado académico de Magíster en Ciencias de la Educación con Mención en Gestión de la Educación. Universidad Nacional Mayor de San Marcos. Lima Perú.

Reyes, N.T. (2012). Liderazgo directivo y desempeño docente en el nivel secundario de una institución educativa de ventanilla callao. Tesis para optar el grado académico de maestro en educación mención en gestión de la educación. Universidad San Ignacio de Loyola. Lima, Perú.

Sum, M. (2015). "Motivación y desempeño laboral (Estudio realizado con el personal administrativo de una empresa de alimentos de la zona 1 de Quetzaltenango)". Tesis para optar el título en el grado de Licenciada. Universidad Rafael Landívar. Quetzaltenango, Guatemala.

Correo electrónico: sebgonz@hotmail.com

Revisión de pares:

Recibido: 31/10/2019

Aceptado: 20/12/2019 